\title{
The evolution and population structure of Lactobacillus fermentum from different naturally fermented products as determined by multilocus sequence typing (MLST)
}

\author{
Tong Dan ${ }^{\dagger}$, Wenjun Liư ${ }^{\dagger}$ Yuqin Song, Haiyan Xu, Bilige Menghe, Heping Zhang and Zhihong Sun ${ }^{*}$
}

\begin{abstract}
Background: Lactobacillus fermentum is economically important in the production and preservation of fermented foods. A repeatable and discriminative typing method was devised to characterize $L$. fermentum at the molecular level. The multilocus sequence typing (MLST) scheme developed was based on analysis of the internal sequence of 11 housekeeping gene fragments (clpX, dnaA, dnaK, groEL, murC, murE, pepX, pyrG, recA, rpoB, and uvrC).

Results: MLST analysis of 203 isolates of L. fermentum from Mongolia and seven provinces/ autonomous regions in China identified 57 sequence types (ST), 27 of which were represented by only a single isolate, indicating high genetic diversity. Phylogenetic analyses based on the sequence of the 11 housekeeping gene fragments indicated that the $L$. fermentum isolates analyzed belonged to two major groups. A standardized index of association $\left(I_{A}{ }^{S}\right)$ indicated a weak clonal population structure in L. fermentum. Split decomposition analysis indicated that recombination played an important role in generating the genetic diversity observed in L. fermentum. The results from the minimum spanning tree strongly suggested that evolution of L. fermentum STs was not correlated with geography or food-type.

Conclusions: The MLST scheme developed will be valuable for further studies on the evolution and population structure of $L$. fermentum isolates used in food products.
\end{abstract}

Keywords: Lactobacillus fermentum, multilocus sequence typing (MLST), Population structure, Traditional Fermented food, Sequence type (ST), Housekeeping gene

\section{Background}

Lactobacillus fermentum is an economically important species of lactic acid bacterium (LAB) used in the production and preservation of fermented food as an acidproducing starter culture [1, 2]. Isolates of L. fermentum originate from a variety of habitats: traditionally fermented milk products, sourdough, fermenting plant materials, faeces and sewage amongst others [3-8]. Lactobacillus fermentum was first described by Beijerink (1901), as an obligate heterofermentative bacterium associated with the fermentation of hexoses to lactic acid $[9,10]$. Another

\footnotetext{
* Correspondence: sunzhihong78@163.com

'Equal contributors

Key Laboratory of Dairy Biotechnology and Engineering, Education Ministry of P. R. China, Department of Food Science and Engineering, Inner Mongolia Agricultural University, Hohhot 010018, P. R. China
}

species, Lactobacillus cellobiosus, which was first described by Rogosa et al., (1953) is also heterofermentative and DNA-DNA hybridization studies showed it to be very similar to $L$. fermentum $[11,12]$. There is now strong evidence to support a close relationship between $L$. cellobiosus and L. fermentum and, in fact, L. cellobiosus has now been reclassified as a biovar of L. fermentum $[5,13]$.

In recent years, molecular typing approaches have been used to characterize L. fermentum and the subspecies within it. For example, L. fermentum isolates could be differentiated from other Lactobacillus species using randomly amplified polymorphic DNA(RAPD-PCR) methods $[14,15]$. RAPD-PCR has also been used in combination with amplified 16S rDNA restriction analysis (16SARDRA), pulsed-field gel electrophoresis with restriction 
fragment length polymorphism (PFGE-RFLP) and ribotyping to characterize 178 isolates from Lactobacillus species in wine [16]. More recently, L. fermentum has been differentiated from $L$. gasseri and $L$. plantarum isolates from the human genital tract using PFGE and fluorescence in situ hybridization (FISH) [17]. However, these methods can sometimes be ambiguous because the majority of bacteria have very similar nutritional requirements and grow under similar environmental conditions [18].

Multilocus sequence typing (MLST), a protocol that was based on partial nucleotide sequences of housekeeping genes, is commonly used to differentiate between isolates of the same microbial species [19]. In recent decades MLST has been developed as a technique to examine the evolution and genetic population structure of bacteria [20-22]. It was initially evaluated for Neisseria meningitidis but has subsequently been extended to many bacterial species [20]. Most recently it has been used to characterize Lactobacillus species including Lactobacillus casei [23, 24], Lactobacillus plantarum [25], Lactobacillus sanfranciscensis [26], Lactobacillus delbrueckii and Lactobacillus sakei [27, 28]. However, until this study, MLST had not been applied to characterizing L. fermentum isolates.

Here we developed an MLST scheme based on 11 housekeeping gene fragments to characterize 203 isolates of $L$. fermentum. The aim of this study was to develop an effective MLST method for L. fermentum and use this to describe the diversity, genetic population structure and evolutionary origins of isolates within this species.

\section{Results}

\section{Sequence diversity in L. fermentum}

Partial sequences of 11 gene fragments $(\operatorname{llp} X, d n a A$, groEL, pyrG, rpoB, recA, murE, pepX, uvrC, dnaK and murC) were determined (Table 1). The numbers of alleles, polymorphic sites, guanine-cytosine content, nucleotide diversity per site $(P i)$ and rate of $d_{N} / d_{S}$ value $\left(d_{S}\right.$ is the number of synonymous substitutions per synonymous site and $d_{N}$ is the number of non-synonymous substitutions per non-synonymous site) were all determined (Table 2). Fragment sizes of the 11 housekeeping gene fragments, which ranged from $589 \mathrm{bp}($ dnaA) to $748 \mathrm{bp}(u v r C)$, were used for MLST analysis. The number of alleles per gene fragment varied between seven ( $g r o E L)$ and 19 (pepX). Between six (groEL) and 22 (pepX) polymorphic sites were found for each gene fragment, and a total of 135 SNPs were identified. The mean guaninecytosine content of the partial sequence of the 11 gene fragments varied between $48.26 \%$ (dnaA) and 60.44\% $(p e p X) . P i$ was calculated for each individual gene fragment and varied from 0.00393 (murC) to 0.01421 (dnaA). The $d_{N} / d_{S}$ value for the 11 gene fragments varied between 0.0000 (clpX, dnaA, dnaK, and recA) and 0.2963 (carB).

\section{Assignment of sequence types}

An MLST protocol was developed for the $203 \mathrm{~L}$. fermentum isolates based on 11 housekeeping gene fragments and used to identify STs (Additional file 1: Table S1). Fiftyseven STs were identified using combined data from the 11 gene fragments (ST-1 to ST-57) (Table S1). The 203 isolates were divided as follows: ST-4 (46 isolates); ST-5 (23 isolates); ST-23 (ten isolates); ST-37 (eight isolates); ST-6, 18 (six isolates each); ST-21, 26, 34, 55 (five isolates each); ST-12, 29, 38 (four isolates each); ST-1, 8, 11, 22, 24, 28, 31, 45, 48, 50, 56 (three isolates each); ST-9, 15, 25, 40, 43, 44 (two isolates each); the remaining 27 STs were each represented by a single isolate.

\section{Relatedness of $L$. fermentum isolates}

To infer the evolutionary relationships amongst $L$. fermentum isolates, a phylogenetic tree based on the MLST data was obtained using the Neighbour-joining (N-J) method (Fig. 1). In the phylogenetic tree built, all of the isolates investigated were well clustered into two major groups, A and B. Group A contained four clonal complexes (CCs, CC2-CC5) and 25 singletons, representing $78 \mathrm{~L}$. fermentem isolates and one reference isolate (ST57); most of the isolates (77 \%) were isolated from acidic gruel. The reference isolate (ST-57), which was also found in Group A, was isolated from fermented plant material. Group B was the larger of the two groups and included $\mathrm{CC} 1$ and nine singletons; almost all the isolates (98\%) were isolated from different dairy products such as yoghurt, kurut, qula, fermented camels' milk, koumiss, whey, etc.. The ancestral type for group B was ST4 , which contained 46 isolates originating from dairy products, in addition to IMAU70163, which was an isolate from acidic gruel.

\section{Recombination in L. fermentum}

The values of $I_{\mathrm{A}}$ (the index of association) and $I_{A}{ }^{\mathrm{S}}$ (the standardised index of association) for the 11 gene fragments were 2.1424 and $0.2142(p=0.000)$, respectively. As the $I_{A}{ }^{\mathrm{S}}$ value was greater than 0 , this indicates that the genes investigated in this study were close to linkage disequilibrium [29].

Simultaneously, a split decomposition analysis examining evidence for recombination amongst the 203 L. fermentum isolates revealed different structures in the split graphs for all 11 gene fragments (Fig. 2). The split graphs for pep $X$, $u v r C$, and $r p o B$ were network-like with parallelogram structures indicative of recombination in the evolutionary history of those genes. However, the split graphs for groEL, dnaK, dnaA, murE, clpX, murC, recA and pyrG were treelike structures, indicative of a clonal descent for these genes and an absence of recombination.

Based on split decomposition analysis the relationships amongst the 57 STs could be described as having a 
Table 1 Genes and primers used for MLST

\begin{tabular}{|c|c|c|c|c|}
\hline Gene & Position* & Primer & Sequence & Amplicon size (bp) \\
\hline \multirow[t]{2}{*}{$\operatorname{clpX}$} & $723485-724143$ & $c l p X / F$ & 5'-CGCACGGAAGCAGAAAC-3' & 659 \\
\hline & & $c l p X / R$ & 5'-GAGTCGGTCCCAAACCC-3' & \\
\hline \multirow[t]{2}{*}{$d n a A$} & 410-998 & $d n a A / F$ & 5'-ACCCGCTCCTGATTTACG-3' & 589 \\
\hline & & $d n a A / R$ & 5'-GCCTCGGTAGCCAGTTTG-3' & \\
\hline \multirow[t]{2}{*}{ dnak } & $885345-886078$ & $d n a K / F$ & 5'-GACAACGGTCCGCTCCACT-3' & 734 \\
\hline & & $d n a k / R$ & 5'-TCGGCTTCTTCCTTCTTCTTCT-3' & \\
\hline \multirow[t]{2}{*}{ groEL } & $394357-395019$ & groEL/F & 5'-CCGACAACGACAAGATGG-3' & 663 \\
\hline & & $\mathrm{groEL} / \mathrm{R}$ & 5'-CCAAGGCAGGGATAACG-3' & \\
\hline \multirow[t]{2}{*}{ murC } & $1501098-1500354$ & murC/F & $5^{\prime}$-TTGAAGCCGACGAATACC - -3' & 745 \\
\hline & & murC/R & 5'-CGATGTCCTCGCTACCC-3' & \\
\hline \multirow[t]{2}{*}{ murE } & 2086362-2085704 & murE/F & 5'-CTACCGCCAGCACTTCTT-3' & 659 \\
\hline & & mUrE/R & 5'-GGTCCATCTGGGTGTTTAGC-3' & \\
\hline \multirow[t]{2}{*}{ pepX } & 1895783-1896506 & pepX/F & 5'-AAAGAAGACGAGCAACCAACC-3' & 724 \\
\hline & & pepX/R & 5'-CGGAGTCCTTAGTCCCGATT-3' & \\
\hline \multirow[t]{2}{*}{ pyrG } & $246712-247420$ & pyrG/F & 5'-TCATTGGGTCGGCTGTT-3' & 709 \\
\hline & & pyrG/R & 5'-GGTCCATCCCTTGCTIITG-3' & \\
\hline \multirow[t]{2}{*}{ recA } & $605296-605942$ & $\operatorname{rec} A / F$ & 5'-ATTGCCGACGCCCTGAT-3' & 647 \\
\hline & & $\operatorname{rec} A / \mathrm{R}$ & 5'-TGCGGTTCGCCTTCCTT-3' & \\
\hline \multirow[t]{2}{*}{$r p o B$} & $1719594-1718880$ & $r p o B / F$ & 5'-GAAGTTCCGCCGCTCTA-3' & 715 \\
\hline & & $r p o B / R$ & 5'-GGTCCCATCTGGCATGTAC-3' & \\
\hline \multirow[t]{2}{*}{ uvrC } & $727309-728056$ & $u v r C / F$ & 5'-TCGTCACCTCCTCCAATAA-3' & 748 \\
\hline & & $u v r C / R$ & 5'-TGGTTCGGTAATCCCTCC-3' & \\
\hline
\end{tabular}

*Positions correspond to complete genome sequence of Lactobacillsu fermentum IFO 3956

Table 2 Nucleotide and allelic diversity in 11 housekeeping gene fragments

\begin{tabular}{lcccll}
\hline Locus & $\begin{array}{l}\text { Number of } \\
\text { Alleles }\end{array}$ & Polymorphic sites & $\begin{array}{l}\text { Mean } G \\
+C \\
\text { content } \\
\text { (mol\%) }\end{array}$ & & \\
\hline clpX & 11 & 11 & 53.24 & 0.00634 & 0 \\
dnaA & 9 & 12 & 48.26 & 0.01421 & 0 \\
dnaK & 12 & 13 & 56.5 & 0.0061 & 0 \\
groEL & 7 & 6 & 51.91 & 0.00416 & 0.2188 \\
murC & 12 & 12 & 55.16 & 0.00393 & 0.0812 \\
murE & 11 & 14 & 54.86 & 0.00768 & 0.0424 \\
pepX & 19 & 22 & 60.44 & 0.00769 & 0.2548 \\
pyrG & 10 & 10 & 54.61 & 0.0056 & 0.128 \\
recA & 12 & 11 & 56.27 & 0.00408 & 0 \\
rpoB & 11 & 7 & 52.63 & 0.00514 & 0.0683 \\
uvrC & 11 & 13 & 52.98 & 0.00618 & 0.1714 \\
\hline
\end{tabular}

${ }^{1}$ Mean pairwise nucleotide differences per site

${ }^{2} d_{N} / d_{S}=$ the ratio of nonsynonymous to synonymous substitutions network-like structure with rays of different lengths (Fig. 3). The STs were divided into two main groups, A and $\mathrm{B}$, and these groups were completely disconnected from each other. Parallelogram-shaped groupings were detected suggesting recombination events had occurred frequently. Isolates in group A were more distantly related to the ancestral isolate than the isolates in group B based on split decomposition analysis, suggesting that recombination had not occurred between isolates from the two groups, but that intergenic recombination may have occurred between isolates from the same group during their evolution. In addition, $57 \mathrm{STs}$ indicated two distinct groups corresponding to group A and B in Fig. 1.

\section{Cluster analysis of the MLST data}

Allelic profile-based phylogenetic analysis using the minimum spanning tree algorithm based on food-type and geographic origins, was used to explore genetic lineages amongst the L. fermentum isolates (Fig. 4). In this representation, isolates with the same allelic profile were assigned to the same circle, the size of the circle is proportional to the number of isolates with that unique profile. The clonal complexes (CCs) were confirmed as 


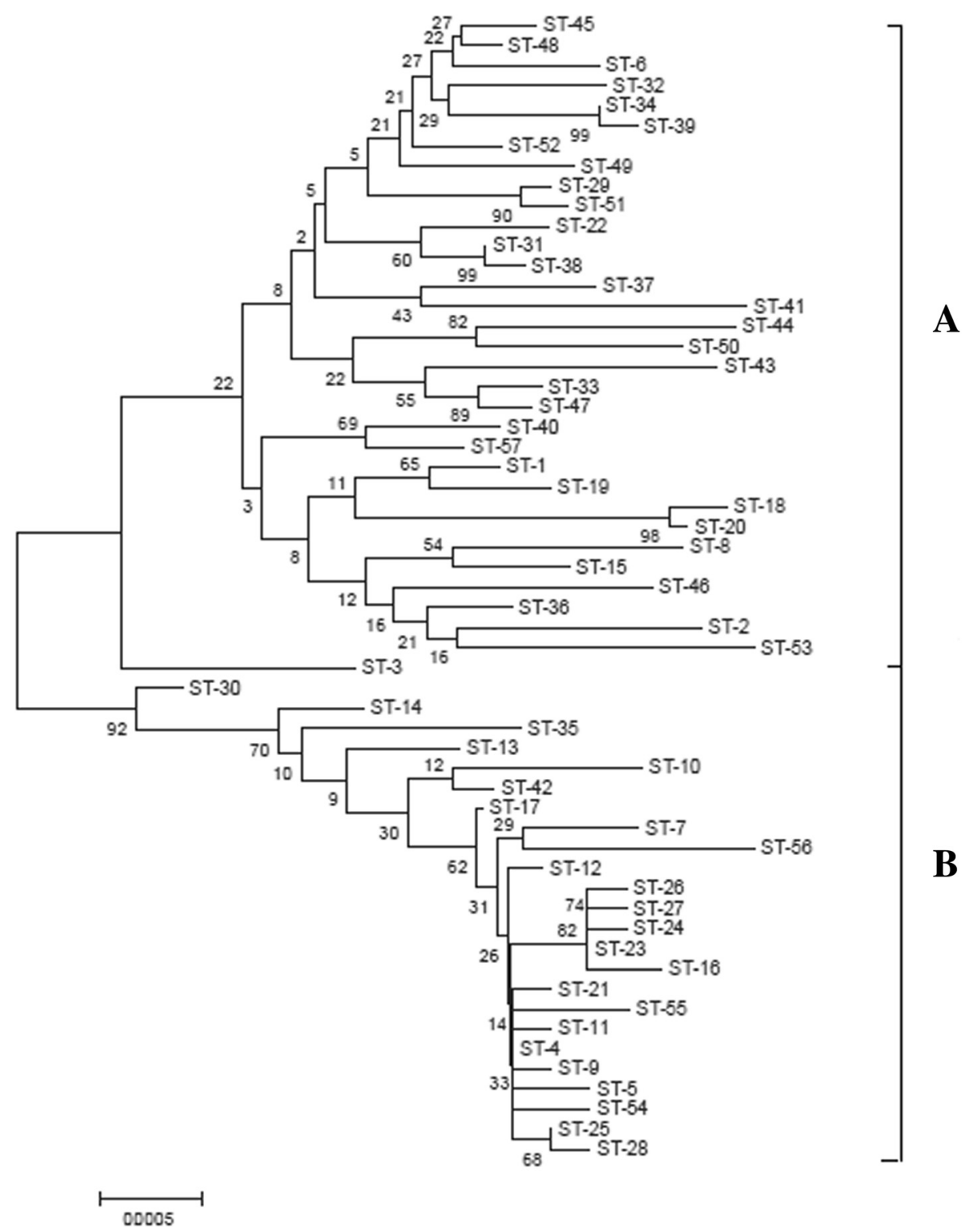

Fig. 1 Neighbour-joining phylogenetic tree obtained from the concatenated nucleotide sequence of 57 STs. Bootstrap value are shown for all branches. The numbering in the figure refers to the ST. The two major phylogroups were designated as A and B

groups of STs sharing 9 of the 11 gene fragments. The 203 L. fermentum isolates evaluated were assigned to 57 STs that were distributed amongst five CCs and 34 unique STs (singletons).

Most of the isolates recovered from acidic gruel were absent from the largest CC, i.e. CC1. CC1 included 15 STs that mainly originated from traditional fermented dairy products from a wide range of geographic locations, including Mongolia, seven regions of Inner Mongolia, Tibet, Gansu, Sichuan, Yunnan, Qinghai and Xinjiang. The only exception was isolate IMAU70163, which belonged to $\mathrm{CC} 1$, but was isolated from acidic gruel. Within CC1, ST-4 had the largest number of isolates $(46=23 \%$ of the total) and was identified as the anscestral genotype surrounded by single-locus (ST-5, 9, $11,12,17,21,23,24,25$ and 27), or two-locus variants (ST-16, 26, 28, and 42). All other CCs included only two STs, each with only a few isolates. CC2 included ST-18 and ST-20, and contained seven isolates from Yunnan. CC3 included ST-31 and ST-38, and contained seven isolates from Inner Mongolia. CC4 included ST-34 and ST-39, and contained six isolates from Inner Mongolia. CC5 included ST-29 and ST-51, and contained five isolates from Tibet and Inner Mongolia. 


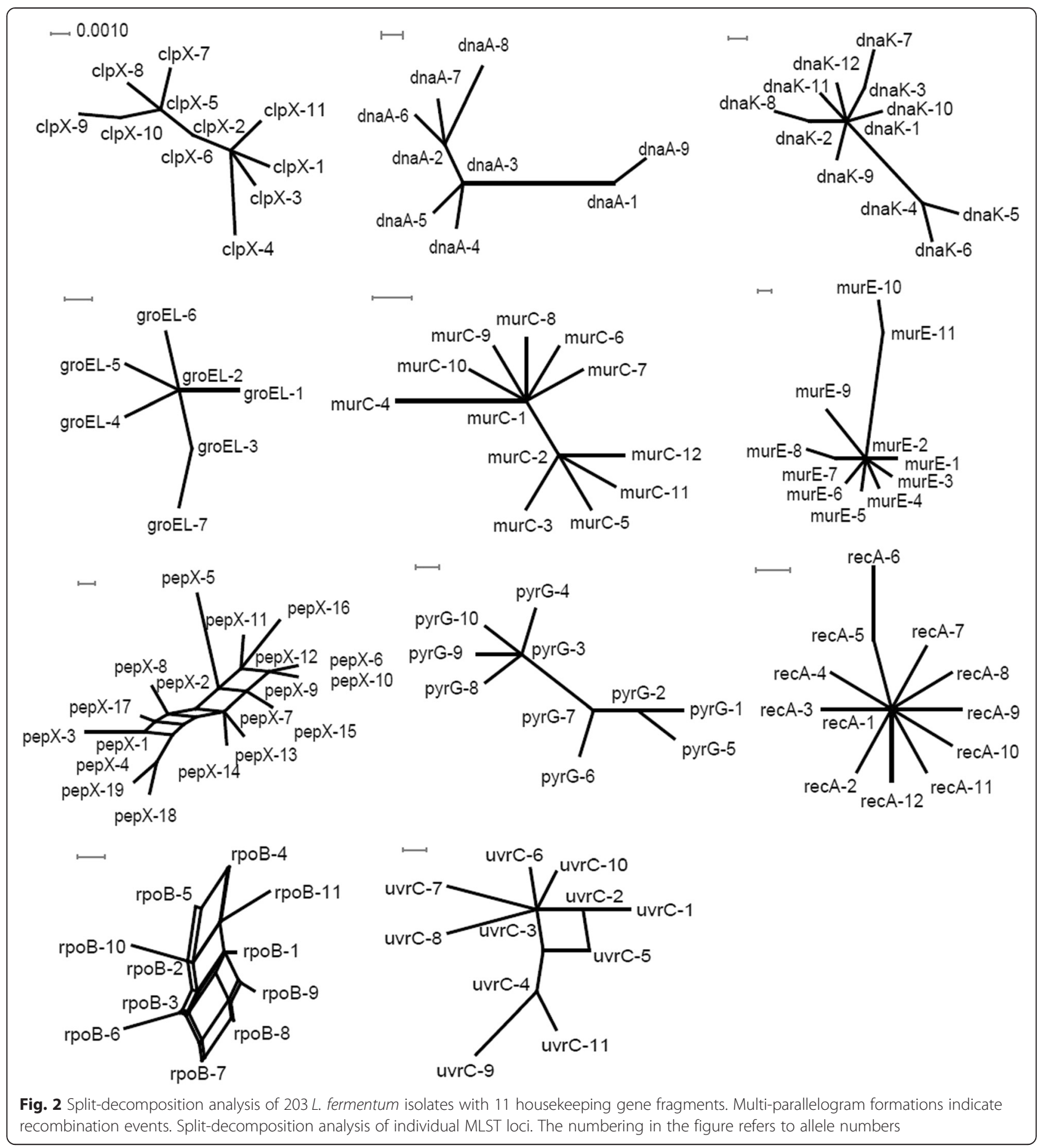

\section{Discussion}

Multilocus sequence typing is considered to be the best method for typing isolates using the DNA sequences of selected housekeeping gene internal fragments [30]. In this study, we used the MLST method to analyze the natural diversity in L. fermentum based on the DNA sequences of 11 housekeeping gene fragments. The isolates evaluated here were isolated from a relatively large geographic area including Mongolia, four Chinese Provinces and two Autonomous regions of China, and from various naturally fermented dairy products and acidic gruel. These isolates provide the relevant information required for a better understanding of the population structure and phylogenetic relationships amongst 203 isolates of $L$. fermentum.

Nucleotide sequences-based methods for bacterial typing are the most unambiguous methods by which isolates 


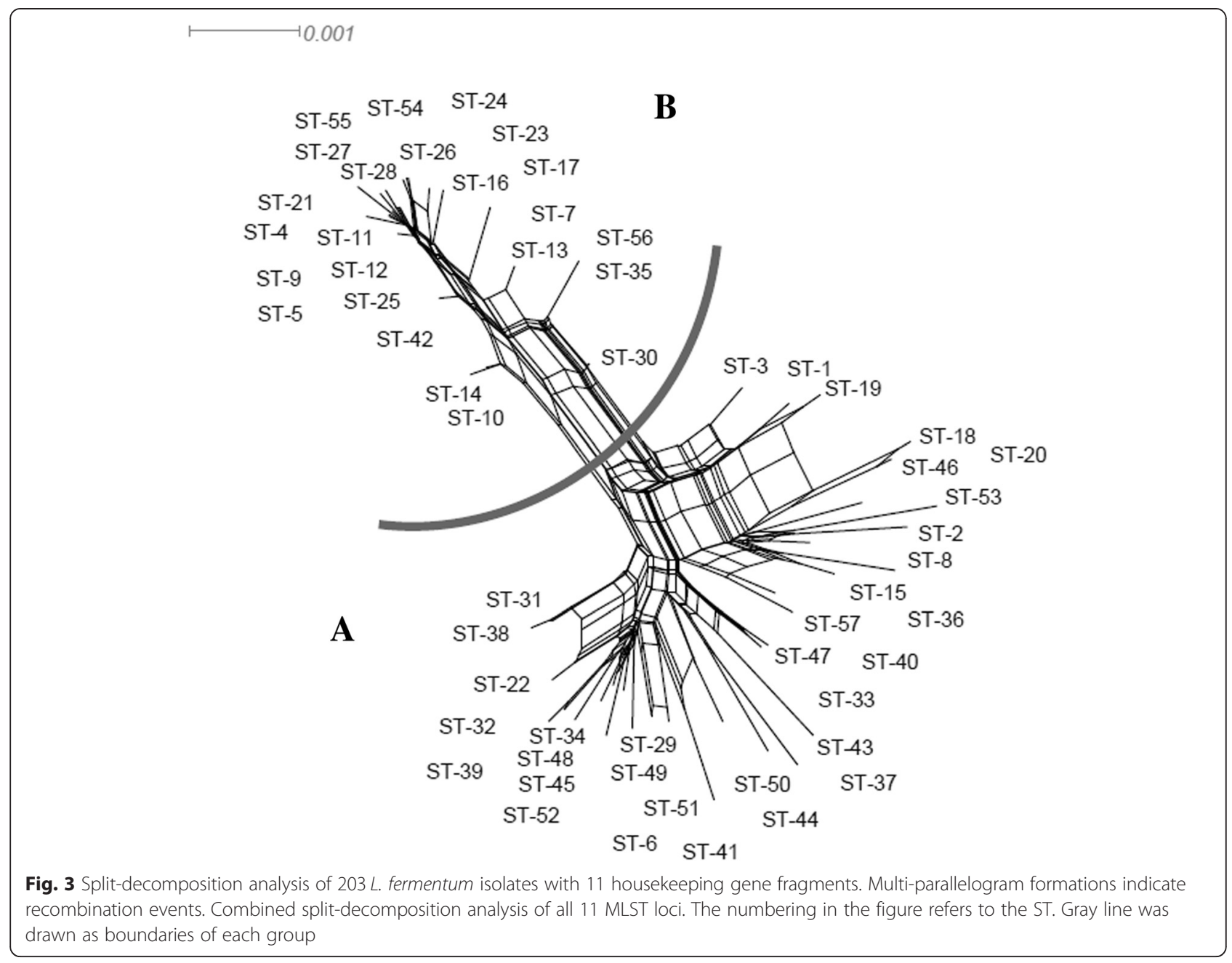

of any microorganism can be identified [30, 31]. In this study, 203 L. fermentum isolates were divided into 57 STs, providing a clear indication of variability. We used the MLST method to compare nucleotide polymorphisms within regions of the 11 housekeeping gene fragments, which are under selective pressure to retain function. Housekeeping genes used in this study had a certain number of polymorphisms and hence were useful for designing the MLST protocol. A total of 131 polymorphic sites were detected in the 11 gene fragments giving a polymorphism rate of $1.72 \%$ amongst the 7,592 nucleotides present in $L$. fermentum isolates. This value was higher than that seen for other LAB, such as Oenococcus oeni, which had 40 SNPs (0.99 \%) amongst 4,040 base pairs sequenced [32]; and other microbes such as Aspergillus fumigatus, with 41 SNPs (1.35 \%) amongst 3,038 base pairs sequenced [33]. This result also indicated that, because most of the gene fragments used in this study had high nucleotide genetic diversity, they also had a strong discriminatory ability.

The value of $P i$ varied from 0.00393 (murC) to 0.01421 $(d n a A)$ for each gene fragment. Except for $d n a A$, most fragments showed similar nucleotide diversity of between 0.00393 and 0.00769 . Our estimate of $P i$ was similar to those obtained for L. plantarum, which ranged from 0.0004 to 0.0072 [25] and $L$. delbrueckii, which ranged from 0.0051 to 0.0096 [27]. These comparisons demonstrate the relatively high nucleotide diversity in these species and in the L. fermentum isolates used in this study. This relatively high nucleotide diversity in L. fermentum is possibly as a result of recombination by natural transformation and a low frequency of mutation. In our genetic analysis, the $d_{N} / d_{S}$ ratios of the 11 gene fragments were all less than 1 , suggesting purifying selection as expected from relatively conserved housekeeping genes. In particular the values for $\operatorname{clp} X, d n a A, d n a K$ and $r e c A$ were zero, indicating that the amino acid composition of these genes did not change. Similar results have also been found in other studies, supporting our conclusion that these housekeeping genes are all under stabilizing selection [34, 35].

The phylogenetic tree constructed from the concatenated sequences of the 11 gene fragments indicated that the 203 L. fermentum isolates formed two distinct groups (A and 


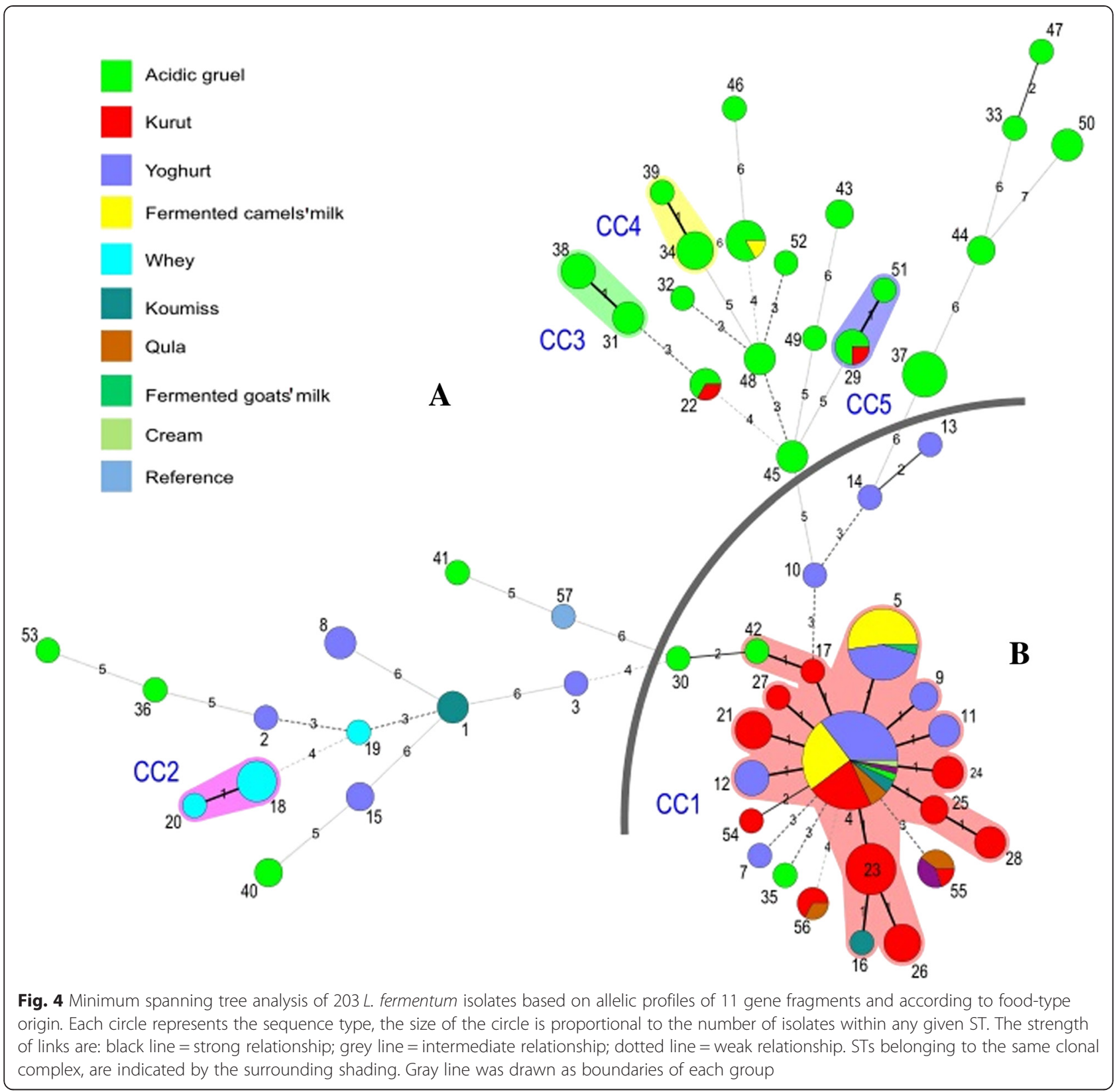

B); partitioning of the two groups was as a result of evolutionary changes in the $\operatorname{clpX}, \operatorname{dnaA}$, dnaK, groEL, murC, murE, pepX, pyrG, recA, rpoB and uvrC gene sequences, as was clearly visible in the N-J phylogenetic tree constructed (Fig. 1). This showed a strong evolutionary tendency for L. fermentum isolates from the same geographic areas, including Mongolia, a number of Chinese Provinces and an Autonomous region, and from the same naturally fermented products, to be similar. MLST analyses were valuable in identifying these differences in variation within the $L$. fermentum genome. Hence, $L$. fermentum isolates that were included in the same group shared similarities in genome organization.
Notably, about eight of ten isolates from acidic gruel were grouped together within group A and almost all the isolates from fermented dairy products were grouped together within group B. The minimum spanning tree analysis supported the existence of two groups of isolates (Fig. 4). Although MLST analysis of 203 L. fermentum isolates indicated two groups here, we cannot exclude the possible that more complex population structures might be identified if a larger number of isolates from more diverse sources was used. In future work, MLST will be a useful tool to examine possible relationships amongst $L$. fermentum isolates, while simultaneously aiding the selection of 
industrial isolates with greatest potential for the production of fermented food.

The analysis of population structure of L. fermentum isolates indicated substantial recombination phenomena. The $I_{A}{ }^{\mathrm{S}}$ value for the 11 gene fragments from $203 \mathrm{~L}$. fermentum isolates was calculated as $0.2142(p=0.000)$, which is indicative of a weak clonal population structure (i.e. linkage disequilibrium). Several studies have already shown that LAB isolated from fermented milk are structured in rather clonal populations, for example, $\mathrm{Xu}$ et al., (2013) investigated 12 housekeeping genes in Lactococcus lactis; the $I_{A}{ }^{\mathrm{S}}$ value was 0.3038 and indicative of a clonal population [35].

Split-decomposition analysis also provided evidence of intraspecies recombination that could play a role in generating genotypic diversity amongst isolates according to the allelic profiles of the isolates evaluated. Split graphs for individual loci indicated tree-like or network-like structures, suggesting that some genes were affected by intraspecies recombination (Fig. 2). Simultaneously, a split graph representation of the concatenated sequence of the 11 loci clearly indicated that two groups and their descents orginated from intraspecies recombination (Fig. 3). The concatenated dendogram corresponded well with the allele-based dendogram (Fig. 1). However, some small differences were found between the concatenated dendogram and the allele-based dendogram. For example, ST-25 was positioned further away from ST-28 in the latter than the former. Although intraspecies recombination occurs frequently, isolates from fermented dairy products in group B and isolates from acidic gruel in group A in both the N-J phylogenetic tree (Fig. 1) and the combined split graph (Fig. 3), seem to be clonal. These results suggest that isolates from fermented dairy products and acidic gruel may have a common recent ancestor. In addition, the minimum spanning tree analysis result confirmed the above assumption that these isolates have a common recent ancestor (Fig. 4).

The clustering of isolates by food-type origin was evident in the minimum spanning tree (Fig. 4) demonstrating strong patterns of specificity for source within the 203 L. fermentum isolates evaluated. The diversity found amongst isolates from fermented dairy products differed from that found amongst isolates from acidic gruel. Almost all of the isolates from fermented dairy products were assigned to the largest $\mathrm{CC}, \mathrm{CC} 1$, whereas isolates from acidic gruel were dispersed among a larger number of STs. Although the difference between isolates from fermented dairy products and acidic gruel was significant, we considered that evolution of $L$. fermentum was not correlated with food-type origins. We suggest that a more simple explanation is that the ecological niches from which the isolates were sampled are very narrow (several types of dairy products and acidic gruels). As L. fermentum is a ubiquitous bacterium found in many different types of food and in animal faeces, it is likely that isolates from dairy products are only a small proportion of the natural diversity within this species, particularly as they would be selected for particular attributes associated with the fermentation of food. We also found no association between STs and geographical origin (data not shown).

MLST data are usually subdivided into nonoverlapping groups of related STs or CCs using an eBURST algorithm approach to determine the most parsimonious patterns of descent of isolates within each $\mathrm{CC}$ from the predicted founder [36]. Here we also used the eBURST algorithm to analyze the MLST data, and found that ST4 inhabited a central location, and that other STs had relatives that were derived from the ubiquitous STs themselves and exhibited a limited genetic diversity, as has been found in other studies [37, 38]. The great majority of STs in CC1 are single-locus variants (SLVs) of ST-4, which strongly supported primary founders. Furthermore, $\mathrm{CC} 1$ to $\mathrm{CC} 5$ were clearly formed based on food-type origins. For example, three of the seven CCs (CC1 to CC2) consisted exclusively of isolates from fermented dairy products, with the exception of only one isolate (IMAU70056), whereas three of five CCs (CC3 to CC5) consisted exclusively of isolates from acidic gruel, with the exception of only one isolate (IMAU60167). Furthermore, we found that the L. fermentum isolates in $\mathrm{CC} 1$, which were almost exclusively isolated from fermented dairy products, were located centrally, with isolates from acidic gruel being distributed around $\mathrm{CC} 1$ and not centrally (Additional file 2: Figure S1).

The genus Lactobacillus, together with the genera Paralactobacillus and Pediococcus, is the largest group in the Lactobacillaceae and order Lactobacillales in the Firmicutes [39]. The species L. fermentum and Lactobacillus reuteri are phylogenetically closely related and are regarded together as the $L$. reuteri group. Since Maiden et al., (1998) first described an MLST technique for $N$. meningitides, MLST methods have been used to differentiate between isolates within species from the genus Lactobacillus including L. casei, L. plantarum, L. sanfranciscensis, L. delbrueckii, and L. sakei, [23-28]. To date, the MLST schemes used in these studies were distinct from each other because the isolates used in each study came from different habitats, and different housekeeping genes were selected for analysis. In these studies, 41 isolates of $L$. delbrueckii were assigned to 34 STs [27]; 52 isolates of $L$. casei (L. paracasei) were assigned to 31 STs [24]; 232 isolates of L. sakei were assigned to 116 unique STs of which all isolates had evolved into three clades, each with a unique population structure [28]. In our study, we used MLST to identify 57 STs within a L. fermentum population of 203 isolates. Our research indicated that this species had a clonal population structure and a pattern of diversity different 
from the previously mentioned species. Moreover, two groups, each with a unique population structure, were identified amongst $L$. fermentum isolates (including the reference isolate $L$. fermentum IFO 3956). The $L$. fermentum MLST schemes in this study demonstrate that MLST is a useful tool for discrimination between isolates, and furthermore provides a method to analyze the evolution and population structure of Lactobacillius species from various sources.

\section{Conclusions}

In this study, a novel MLST protocol was used to investigate the population genetic structure and evolutionary characteristics of L. fermentum. The MLST protocol presented provided high discriminatory power for molecular typing of L. fermentum isolates. Furthermore, we were able to shed light on how this species has evolved into two unique groups. Using a large number of isolates allowed us to better interpret the possible ecological differences underlying the two branches observed. Finally, we also found that the evolution of $L$. fermentum STs was not correlated with geography or food-type origin. Taken together, our results indicate that MLST of L. fermentum was an easy and valuable tool that, together with the construction of an MLST database, will contribute to further detailed studies on the evolution and population genetics of $L$. fermentum.

\section{Methods}

\section{Bacterial isolates and growth conditions}

A total of $203 \mathrm{~L}$. fermentum isolates, from the Lactic Acid Bacteria Collection Center of the Inner Mongolia Agricultural University (LABCC), were used in this study (Table S1). These isolates came from various sources including yoghurt, kurut, koumiss, fermented camels' milk, fermented goats' milk, qula, whey, cream, acidic gruel and other traditional foods from Mongolia; the Provinces of Sichuan, Qinghai, Yunnan and Gansu from the P.R of China; and the Autonomous Regions of Inner Mongolia, Xinjiang and Tibet from the P.R of China. All isolates were cultured under anaerobic conditions in de Man Rogosa Sharpe broth (MRS) (Becton, Dickinson Co., Sparks, Md., USA) at $37^{\circ} \mathrm{C}$ for $24 \mathrm{~h}$. The reference isolate, L. fermentum IFO 3956 which was originally obtained from fermented plant material, was obtained from the NCBI genome database (http://www.ncbi.nlm.nih.gov/genome/).

\section{DNA extraction}

Bacterial DNA was extracted from all isolates of L. fermentum as described previously [40]. Briefly, the bacterial cells were precipitated out of suspension by centrifugation $\left(8,000 \times \mathrm{g}, 3 \mathrm{~min}, 4^{\circ} \mathrm{C}\right)$ after overnight incubation in MRS broth at $37^{\circ} \mathrm{C}$. The pellet was subjected to freeze-thaw cycles to ensure cell lysis. After thoroughly washing the pellet with phosphate-buffered saline (PBS), $10 \%$ SDS and proteinase-K solution $(20 \mathrm{mg} / \mathrm{ml})$ were added and incubated in a shaking incubator at $200 \mathrm{rpm}$ and $37^{\circ} \mathrm{C}$ overnight. Subsequently, $0.7 \mathrm{M} \mathrm{NaCl}$ and $10 \%$ cetyl trimethyl ammonium bromide (CTAB) were added and incubated at $65^{\circ} \mathrm{C}$ for a further 20 minutes. Protein contaminants were removed by phenol extraction and the DNA was precipitated with an equal volume of ice-cold isopropanol, and then washed thoroughly in $70 \%(\mathrm{v} / \mathrm{v})$ ice-cold ethanol. The final DNA concentration was determined by recording its optical density at 260 and $280 \mathrm{~nm}$, respectively, using a NanoDrop ND-1000 spectrophotometer (NanoDrop Technologies, Wilmington, DE, USA).

\section{Selection of housekeeping genes for MLST}

General criteria for selection of housekeeping genes include their location on the chromosome, the function of the encoded proteins, their presence in all isolates as a single copy and that gene size is at least $1 \mathrm{~kb}$ [23]. Based on these criteria and by examining the gene sequences of $L$. fermentum IFO 3956, 11 housekeeping genes (clpX, dnaA, dnaK, groEL, murC, murE, pepX, pyrG, recA, $r p o B$ and $u v r C$ ) were selected. Among them, ten housekeeping genes (clpX, dnaA, dnaK, groEL, murC, murE, pepX, pyrG, recA and $u v r C$ ) were selected based on the results of a previous study on Lactobacillus helveticus [41], the remaining locus (rpoB) was based on Lactobacillus sakei [28].

These housekeeping genes were also described from the variable regions in the L. fermentum IFO 3956 genome sequence [42]: clpX encoding ATP-dependent protease ATP-binding subunit ClpX (YP_001843436), dnaA encoding chromosomal replication initiation protein (YP_001 842817.1), groEL encoding chaperonin GroEL (YP_00 1843142.1), pyrG encoding CTP synthetase (YP_001 843018.1), rpoB encoding DNA-directed RNA polymerase subunit beta (YP_001844339.1), recA encoding recombinase A (YP_001843327.1), murE encoding UDP-Nacetylmuramoylalanyl-D-glutamate-2, 6-diaminopimelate ligase (YP_001844650.1), pepX encoding x-prolyl-dipeptidyl aminopeptidase (YP_001844484.1), uvrC encoding excinuclease ABC subunit C (YP_001843441.1), dnaK encoding molecular chaperone DnaK (YP_001843567.1) and murC encoding UDP-N-acetylmuramate-L-alanine ligase (YP_001844136.1).

\section{PCR amplification and nucleotide sequencing}

In this study, the MLST protocol was modified to study the population structure of L. fermentum using the 11 housekeeping genes selected. Bacterial DNA from the 203 isolates of $L$. fermentum was used as the template for amplification of the 11 housekeeping genes. Primers were designed based on the internal fragments of the 11 gene fragments with Primer Premier 6.0 software 
(Table 1). Primers targeting the internal fragments of each gene ranged from 589 to $748 \mathrm{bp}$ in size. The PCR mixture $(10 \mu \mathrm{l})$ contained $0.08 \mu \mathrm{l} \mathrm{Taq}$ polymerase $(5 \mathrm{U} / \mu \mathrm{l}$, Takara, Tokyo), $1 \mu \mathrm{l} 10 \times \mathrm{PCR}$ buffer $\left(\mathrm{Mg}^{2+}\right.$ free), $0.8 \mu \mathrm{l}$ dNTPs (2.5 mM each), $0.8 \mu \mathrm{MgCl}_{2}(25 \mathrm{mM}), 0.4 \mu \mathrm{l}$ forward primer $(10 \mu \mathrm{M}), 0.4 \mu \mathrm{l}$ reverse primer $(10 \mu \mathrm{M}), 1 \mu \mathrm{l}$

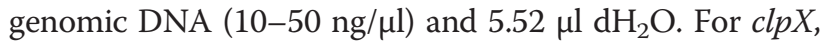
dnaA, groEL, pyrG, rpoB, recA, murE, pepX uvrC, dnaK and $m u r C$, the PCR procedure was done under the following conditions: $94^{\circ} \mathrm{C}$ for $5 \mathrm{sec}, 30$ cycles of amplification which included $95^{\circ} \mathrm{C}$ for $60 \mathrm{sec}, 50^{\circ} \mathrm{C}$ for $45 \mathrm{sec}, 72^{\circ} \mathrm{C}$ for $60 \mathrm{sec}$ and then annealing at $72{ }^{\circ} \mathrm{C}$ for $10 \mathrm{~min}$. Sequencing of the PCR products was done by the Shanghai Sangni Biosciences Corporation (Shanghai, China). Every isolate was sequenced by Sanger dideoxy sequencing, using both DNA strands. Each MLST allele fragment was sequenced on both nucleotide strands. After obtaining sequences for each isolate, we trimmed, aligned and adjusted these sequences using the MEGA 5.0 software package, and these sequences had forward and reverse sequences, which guaranteed their validity.

\section{Descriptive analysis of MLST sequence data}

The sequence data obtained for the 11 housekeeping gene fragments were imported into BioNumerics software (version 6.0, Applied-Maths, Sint Maartens-Latem, Belgium) and allele numbers per gene fragment were obtained. Afterwards, the combination of 11 allele numbers per isolate was assigned to an allelic profile, i.e. a sequence type [43]. Isolates with the same ST had identical allelic profiles. To analyze the micro-evolutionary processes linking STs from all isolates, a minimum-spanning tree was constructed with Prims's algorithm in the BioNumerics software according to region and source separation (version 6.0, Applied-Maths, Sint Maartens-Latem, Belgium).

The guanine-cytosine content, $d_{N} / d_{S}$ ratio, nucleotide diversity, the number of polymorphic sites and single nucleotide polymorphisms (SNPs) in the 11 housekeeping gene fragments for each isolate, were calculated using DnaSP $5.0[44,45]$ and START 2.0 (http://www.mlst.net/ links/software.asp) [46].

Phylogenetic trees were constructed using the neighbourjoining method in MEGA version 5.0 software (version 5.0, www.megasoftware.net). Identifying the relationships between individual STs and their clustering into clonal complexes was achieved using eBURST (Based Upon Related Sequence Types) V 3.0 software (http://eburst.mlst.net) in relation to their number of SLVs, double-locus variants (DLVs) or triple-locus variants (TLVs) [30, 37]. Split decomposition analysis was done with SplitsTree 4.0 and START 2.0 software on the MLST website (http://eburst.mlst.net/) [37]. The level of linkage disequilibrium between the 11 alleles of 203 isolates was calculated in START 2.0 software by determining the value of $I_{A}{ }^{\mathrm{S}}$ [47].

\section{Nucleotide sequence accession numbers}

The partial sequences of the 11 MLST loci used in this study have been deposited in the GenBank/EMBL databases under accession numbers KR078446-KR080061 and KP224504-KP225109.

\section{Additional files}

\begin{abstract}
Additional file 1: Table S1. Allelic profiles of 203 Lactobacillus fermentum isolates. The information on isolates used in this study are listed and identified.

Additional file 2: Figure S1. eBURST analysis of 203 L. fermentum isolates used in this study. In the eBURST diagram, five clonal and thirty-four singletons are shown. The primary founders of eBURST groups are positioned centrally in the cluster and labeled in blue, and the subgroup founders are shown in yellow. Dots indicate sequence type and lines connect single-locus variants, which are STs that differ in only one of the 11 housekeeping gene fragments. Boxed numbers indicate STs found in acidic gruel, the other numbers indicated STs found in dairy products.
\end{abstract}

\section{Abbreviations}

CCs: Clonal Complexes; $d_{N}$ : Non-synonymous Substitutions; $d_{s}$ : Synonymous Substitutions; FISH: Fluorescence in Situ Hybridization; $I_{A}$ : Standardized Index of Association; LAB: Lactic Acid Bacterium; MLST: Multilocus Sequence Typing; PFGE-RFLP: Pulsed-field Gel Electrophoresis with Restriction Fragment Length Polymorphism; RAPD-PCR: Randomly Amplified Polymorphic DNA; SNP: single nucleotide polymorphism; ST: Sequence Type..

\section{Competing interests}

The authors declare that they have no competing interests.

\section{Authors' contributions}

Conceived and designed the experiments: TD ZHS WJL HPZ. Performed the experiments: HYX YQS. Analyzed the data: ZHS HM YQS. Contributed reagents/materials/analysis tools: ZHS HYX YQS. Wrote the paper: TD HPZ. All authors read and approved the final manuscript.

\section{Acknowledgements}

The National Natural Science Foundation of China(Grant No.31460446, Hi-Tech Research and Development Program of China (863 Planning, Grant No. 2011AA100901, 2011AA100902), International S\&T Cooperation Program of China (ISTCP, Grant No.2014DFR31150), the Open Projects of Inner Mongolia Natural Science Foundation (No. 20102010), the Natural Science Foundation of Inner Mongolia (No. 2012MS0507).

Received: 12 January 2015 Accepted: 14 May 2015 Published online: 20 May 2015

\section{References}

1. Tulumoğlu Ş, Kaya Hi, Şimşek Ö. Probiotic characteristics of Lactobacillus fermentum strains isolated from tulum cheese. Anaerobe. 2014;30C:120-5.

2. Swain MR, Anandharaj M, Ray RC, Parveen Rani R. Fermented fruits and vegetables of Asia: a potential source of probiotics. Biotechnol Res Int. 2014;2014:250424.

3. Abdelgadira WS, Hamadb SH, Moller PL, Jakobsen M. Characterization of the dominant microbiota of Sudanese fermented milk Rob. Int Dairy J. 2001;11:63-70

4. Dan T, Fukuda K, Sugai-Bannai M, Takakuwa N, Motoshima H, Urashima T. Characterization and expression analysis of the exopolysaccharide gene cluster in Lactobacillus fermentum TDS030603. Biosci, Biotechnol, Biochem. 2009;73(12):2656-64.

5. Dellaglio F, Torriani S, Felis GE. Reclassification of Lactobacillus cellobiosus Rogosa et al. 1953 as a later synonym of Lactobacillus fermentum Beijerinck. Int J Syst Evol Microbiol 2004. 1901;54(3):809-12.

6. Gil-Campos M, López MÁ, Rodriguez-Benítez MV, Romero J, Roncero I, Linares MD, et al. Lactobacillus fermentum CECT 5716 is safe and well 
tolerated in infants of 1-6 months of age: a randomized controlled trial. Pharmacol Res. 2012;65(2):231-8.

7. Fazeli MR, Shahverdi AR, Sedaghat B, Amalifar H, Samadi N. Sourdoughisolated Lactobacillus fermentum as a potent anti-mould preservative of a traditional Iranian bread. Eur Food Res Technol. 2004;218:554-6.

8. Morita H, Yoshikawa H, Sakata R, Nagata $Y$, Tanaka H. Synthesis of nitric oxide from the two equivalent guanidino nitrogens of L-arginine by Lactobacillus fermentum. J Bacteriol. 1997;179(24):7812-5.

9. Beijerink MW. Sur les ferments lactiques de l'industrie. Arch Ne'erl Sci Exactes Nat Se'rie II. 1901;7:212-43.

10. Stiles ME, Holzapfel WH. Lactic acid bacteria of foods and their current taxonomy. Int J Food Microbiol. 1997;36(1):1-29.

11. Rogosa M, Wiseman RF, Mitchell JA, Disraely MN, Beaman AJ. Species differentiation of oral lactobacilli from man including description of Lactobacillus salivarius nov spec and Lactobacillus cellobiosus nov spec. J Bacteriol. 1953;65(6):681-99.

12. Vescovo M, Dellaglio F, Bottazzi V, Sarra PG. Deoxyribonucleic acid homology among Lactobacillus species of the subgenus Betabacterium Orla-Jensen. Microbiologica. 1979;2:317-30.

13. Collins MD, Rodrigues UM, Ash C, Aguirre M, Farrow JAE, Martinez-Murcia A, et al. Phylogenetic analysis of the genus Lactobacillus and related lactic acid bacteria as determined by reverse transcriptase sequencing of 165 rRNA. FEMS Microbiol Lett. 1991;77:5-12.

14. Gardiner GE, Heinemann C, Bruce AW, Beuerman D, Reid G. Persistence of Lactobacillus fermentum RC-14 and Lactobacillus rhamnosus GR-1 but not L. rhamnosus GG in the human vagina as demonstrated by randomly amplified polymorphic DNA. Clin Diagn Lab Immunol. 2002;9(1):92-6.

15. Nigatu A, Ahrné S, Molin G. Randomly amplified polymorphic DNA (RAPD) profiles for the distinction of Lactobacillus species. Antonie Van Leeuwenhoek. 2001;79(1):1-6.

16. Rodas AM, Ferrer S, Pardo I. Polyphasic study of wine Lactobacillus strains: taxonomic implications. Int J Syst Evol Microbiol. 2005;55(1):197-207.

17. Gosiewski T, Chmielarczyk A, Strus M, Brzychczy-Włoch M, Heczko PB. The application of genetics methods to differentiation of three Lactobacillus species of human origin. Ann Microbiol. 2012;62(4):1437-45.

18. Vandamme P, Pot B, Gillis M, De Vos P, Kersters K, Swings J. Polyphasic taxonomy, a consensus approach to bacterial systematics. Microbiol Rev. 1996;60(2):407-38

19. Bain JM, Tavanti A, Davidson AD, Jacobsen MD, Shaw D, Gow NA, et al. Multilocus sequence typing of the pathogenic fungus Aspergillus fumigatus. J Clin Microbiol. 2007;45(5):1469-77.

20. Maiden MC, Bygraves JA, Feil E, Morelli G, Russell JE, Urwin R, et al Multilocus sequence typing: a portable approach to the identification of clones within populations of pathogenic microorganisms. Proc Natl Acad Sci U S A. 1998:95(6):3140-5.

21. Feil EJ, Enright MC. Analyses of clonality and the evolution of bacterial pathogens. Curr Opin Microbiol. 2004;7(3):308-13.

22. Oh PL, Benson AK, Peterson DA, Patil PB, Moriyama EN, Roos S, et al. Diversification of the gut symbiont Lactobacillus reuteri as a result of host-driven evolution. ISME J. 2010;4(3):377-87.

23. Cai H, Rodriguez BT, Zhang W, Broadbent JR, Steele JL. Genotypic and phenotypic characterization of Lactobacillus casei strains isolated from different ecological niches suggests frequent recombination and niche specificity. Microbiology. 2007;153(8):2655-65.

24. Diancourt L, Passet V, Chervaux C, Garault P, Smokvina T, Brisse S. Multilocus sequence typing of Lactobacillus casei reveals a clonal population structure with low levels of homologous recombination. Appl Environ Microbiol. 2007;73(20):6601-11.

25. de Las RB, Marcobal A, Muñoz R. Development of a multilocus sequence typing method for analysis of Lactobacillus plantarum strains. Microbiology. 2006;152(1):85-93.

26. Picozzi C, Bonacina G, Vigentini I, Foschino R. Genetic diversity in Italian Lactobacillus sanfranciscensis strains assessed by multilocus sequence typing and pulsed-field gel electrophoresis analyses. Microbiology. 2010;156:2035-45.

27. Tanigawa K, Watanabe K. Multilocus sequence typing reveals a novel subspeciation of Lactobacillus delbrueckii. Microbiology. 2011;157(3):727-38.

28. Chaillou S, Lucquin I, Najjari A, Zagorec M, Champomier-Vergès MC. Population genetics of Lactobacillus sakei reveals three lineages with distinct evolutionary histories. PLoS One. 2013;8(9):e73253.

29. Feil EJ, Cooper JE, Grundmann H, Robinson DA, Enright MC, Berendt T, et al. How clonal is Staphylococcus aureus? J Bacteriol. 2003;185:3307-16.
30. Urwin R, Maiden MC. Multi-locus sequence typing: a tool for global epidemiology. T rends Microbiol. 2003:11(10):479-87.

31. Cooper JE, Feil EJ. Multilocus sequence typing-what is resolved? Trends Microbiol. 2004;12(8):373-7.

32. de Las RB, Marcobal A, Muñoz R. Allelic diversity and population structure in Oenococcus oeni as determined from sequence analysis of housekeeping genes. Appl Environ Microbiol. 2004;70(12):7210-9.

33. Bain JM, Tavanti A, Davidson AD, Jacobsen MD, Shaw D, Gow NA, et al. Multilocus sequence typing of the pathogenic fungus Aspergillus fumigatus. J Clin Microbiol. 2007:45(5):1469-77.

34. Madslien EH, Olsen JS, Granum PE, Blatny JM. Genotyping of B. licheniformis based on a novel multi-locus sequence typing (MLST) scheme. BMC Microbiol. 2012;12:230.

35. Xu H, Sun Z, Liu W, Yu J, Song Y, Lv Q, et al. Multilocus sequence typing of Lactococcus lactis from naturally fermented milk foods in ethnic minority areas of China. J Dairy Sci. 2014;97(5):2633-45.

36. Feil EJ, Li BC, Aanensen DM, Hanage WP, Spratt BG. eBURST: inferring patterns of evolutionary descent among clusters of related bacterial genotypes from multilocus sequence typing data. J Bacteriol. 2004;186(5):1518-30.

37. Huson DH. SplitsTree: analyzing and visualizing evolutionary data. Bioinformatics. 1998;14(1):68-73.

38. Enright MC, Robinson DA, Randle G, Feil EJ, Grundmann H, Spratt BG. The evolutionary history of methicillin-resistant Staphylococcus aureus (MRSA). Proc Natl Acad Sci U S A. 2002:99(11):7687-92.

39. Felis GE, Dellaglio F. Taxonomy of Lactobacilli and Bifidobacteria. Curr Issues Intest Microbiol. 2007;8(2):44-61.

40. Dan T, Chen X, Bao QH, Liu WJ, Zhang HP. Effect of L-Threonine concentrations on acetaldehyde production and glyA gene expression in fermented milk by Streptococcus thermophilus. Food Biotechnol. 2012;26(3):280-92.

41. Sun ZH, Liu WJ, Song YQ, Xu HY, Yu J, Menghe B, Zhang HP, Chen YF: Population structure of Lactobacillus helveticus isolates from naturally fermented dairy products based on multilocus sequence typing. J dairy Sci 2015, DOl: http://dx.doi.org/10.3168/jds.2014-9133.

42. Morita H, Toh H, Fukuda S, Horikawa H, Oshima K, Suzuki T, et al. Comparative genome analysis of Lactobacillus reuteri and Lactobacillus fermentum reveal a genomic island for reuterin and cobalamin production. DNA Res. 2008;15(3):151-61.

43. Bilhère $E$, Lucas PM, Claisse $O$, Lonvaud-Funel A. Multilocus sequence typing of Oenococcus oeni: detection of two subpopulations shaped by intergenic recombination. Appl Environ Microbiol. 2009;75(5):1291-300.

44. Rozas J, Sánchez-DelBarrio JC, Messeguer X, Rozas R. DnaSP, DNA polymorphism analyses by the coalescent and other methods. Bioinformatics. 2003;19(18):2496-7.

45. Librado P, Rozas J. DnaSP v5: a software for comprehensive analysis of DNA polymorphism data. Bioinformatics. 2009;25(11):1451-2.

46. Jolley KA, Feil EJ, Chan MS, Maiden MC. Sequence type analysis and recombinational tests (START). Bioinformatics. 2001;17(12):1230-1.

47. Suerbaum S, Lohrengel M, Sonnevend A, Ruberg F, Kist M. Allelic diversity and recombination in Campylobacter jejuni. J Bacteriol. 2001;183(8):2553-9.

\section{Submit your next manuscript to BioMed Central and take full advantage of:}

- Convenient online submission

- Thorough peer review

- No space constraints or color figure charges

- Immediate publication on acceptance

- Inclusion in PubMed, CAS, Scopus and Google Scholar

- Research which is freely available for redistribution 